\title{
Surface heating of wire plasmas using laser-irradiated cone geometries
}

\author{
J. S. GREEN ${ }^{1,2}$, K. L. LANCASTER ${ }^{1}$, K. U. AKLI ${ }^{3,4}$, C. D. GREGORY ${ }^{5}$, F. N. BEG ${ }^{6}$, S. N. CHEN ${ }^{6}$, D. CLARK ${ }^{7}$, \\ R. R. FREEMAN7 ${ }^{7}$, S. HAWKES ${ }^{1}$, C. HERNANDEZ-GOMEZ1 ${ }^{1}$, H. HABARA ${ }^{8}$, R. HEATHCOTE1 , D. S. HEY ${ }^{3,4}$, \\ K. HIGHBARGER ${ }^{7}$, M. H. KEY ${ }^{3}$, R. KODAMA ${ }^{8,9}$, K. KRUSHELNICK ${ }^{10}$, I. MUSGRAVE1 , H. NAKAMURA ${ }^{9}$, \\ M. NAKATSUTSUMI ${ }^{8}$, N. PATEL ${ }^{4}$, R. STEPHENS ${ }^{11}$, M. STORM ${ }^{12}$, M. TAMPO ${ }^{9}$, W. THEOBALD ${ }^{12}$, \\ L. VAN WOERKOM7 ${ }^{7}$, R. L. WEBER ${ }^{7}$, M. S. WEl6,11, N. C. WOOLSEY5 AND P. A. NORREYS ${ }^{1,2 *}$ \\ ${ }^{1}$ STFC Rutherford Appleton Laboratory, Chilton OX11 0QX, UK \\ ${ }^{2}$ Blackett Laboratory, Imperial College London, Prince Consort Road, London SW7 2BZ, UK \\ ${ }^{3}$ Lawrence Livermore National Laboratory, PO Box 808, Livermore, California 94550, USA \\ ${ }^{4}$ Department of Applied Sciences, University of California, 1 Shields Avenue, Davis, California 95616-8254, USA \\ ${ }^{5}$ Department of Physics, University of York, Heslington, York Y010 5DD, UK \\ ${ }^{6}$ Department of Mechanical and Aerospace Engineering, University of California San Diego, 9500 Gilman Drive 0411, La Jolla, California 92093-0411, USA \\ ${ }^{7}$ Department of Physics, Ohio State University, Columbus, Ohio, Ohio 43210-1117, USA \\ ${ }^{8}$ Graduate School of Engineering, Osaka University, Suita, 565-0871 Osaka, Japan \\ ${ }^{9}$ Institute of Laser Engineering, Osaka University, Suita, 565-0871 Osaka, Japan \\ ${ }^{10}$ Department of Electrical Engineering and Computer Science, University of Michigan, 1301 Beal Avenue, Ann Arbor, Michigan 48109-2122, USA \\ ${ }^{11}$ General Atomics, PO Box 86508, San Diego, California 92186-5608, USA \\ ${ }^{12}$ Laboratory of Laser Energetics, University of Rochester, 250 East River Road, Rochester, New York 14623, USA \\ *e-mail:p.norreys@rl.ac.uk
}

Petawatt lasers can generate extreme states of matter, making them unique tools for high-energy-density physics. Pressures in the gigabar regime can potentially be generated with cone-wire targets when the coupling efficiency is high and temperatures reach $2-4 \mathrm{keV}$ (ref. 1). The only other method of obtaining such gigantic pressures is to use the megajoule laser facilities being constructed (National Ignition Facility and Laser MégaJoule). The energy can be transported over surprisingly long distances but, until now, the guiding mechanism has remained unclear. Here, we present the first definitive experimental proof that the heating is maximized close to the wire surface, by comparison of interferometric measurements with hydrodynamic simulations. New hybrid particle-in-cell simulations show the complex field structures for the first time, including a reversal of the magnetic field on the inside of the wire. This increases the return current in a spatially separated thin layer below the wire surface, resulting in the enhanced level of ohmic heating. There are a significant number of applications in high-energy-density science, ranging from equation-of-state studies to bright, hard X-ray sources, that will benefit from this new understanding of energy transport.

Cone-wire geometries offer a simpler test case to study energy coupling for fast-ignition inertial fusion ${ }^{2-5}$ and have other applications for high-energy-density plasma science, as described above. The geometry itself is quasi-one-dimensional in nature and therefore avoids the full complexity of a laser-solid-density foilplasma interaction. Above all, the geometry allows fast electron energy transport to be studied without the complications that arise from a (usually divergent) fast electron beam. This is a complex combination of the laser pulse interaction in the coronal plasma, the electric and magnetic fields associated with the propagation of the fast electrons and the return current in the overdense plasma and beam-plasma instabilities that arise as a result ${ }^{6-11}$.

Here, experiments are reported for cone-attached target geometries that have intensities on target of $4 \times 10^{20} \mathrm{~W} \mathrm{~cm}^{-2}$, more than an order of magnitude higher than any previously reported study. A number of complementary measurement techniques were used to diagnose the interaction, including shadowgraphy ${ }^{12}$, interferometry and a number of X-ray imaging techniques, including $\mathrm{K} \alpha \mathrm{X}$-ray imaging ${ }^{13}$. The interferograms clearly show that the energy absorption is maximized close to the tip of the cone. Density profiles, unfolded from the interferometric measurements, indicate that a $7 \mu \mathrm{m}$ copper wire plasma reaches a maximum initial temperature of $400 \mathrm{eV}$ at the wire surface, by comparison with hydrodynamic simulations. It is found that the density profiles can only be reproduced provided that there is an initial non-uniform temperature profile on the wire, with the hottest region located on the outer surface. Hybrid particle-in-cell (PIC) simulations show a fast electron flow along the wire and a compensating return current just inside the wire surface, leading to a greater degree of ohmic heating.

Figure 1 shows a cone-wire interferogram of the Osaka University cone-wire target that had an initial wire diameter of $7 \mu \mathrm{m}$ (see the Methods section for target details). Similar interferograms were obtained for the other targets that were irradiated. The initial target position is shown as a dotted line to guide the eye. It should be noted that the apparent radial 


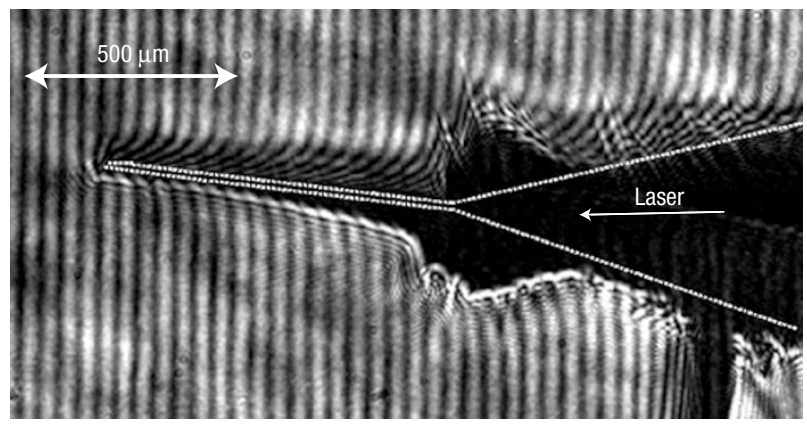

Figure 1 Interferogram of a cone-wire target that was used to calculate plasma densities from the observed phase shifts. The initial wire diameter was $7 \mu \mathrm{m}$ and the image was taken $400 \mathrm{ps}$ after the main interaction.

asymmetry in Fig. 1 is due to an optical misalignment that had no effect on the measured densities, but did reduce the density cutoff on one side of the wire. The observable density profiles from each side were found to be consistent with each other and so for subsequent analysis the side of the wire with the more complete fringe shift was used.

These interferograms all showed that the tip of the cone has exploded radially much faster than the attached wires. The differences in the glue joints strongly affect the energy coupling to the wire plasma, but do not change the overall expansion profile around the cone tip. At distances of less than $200 \mu \mathrm{m}$ from the cone tip, it is not possible to make density measurements of the wire material owing to the presence of the surrounding cone assembly which is also expanding. X-ray imaging techniques ${ }^{13}$ are able to provide more precise information on the degree of energy coupling into the wire in this region as $\mathrm{Cu} \mathrm{K} \alpha$ imaging is able to distinguish between the two heated materials. Here, energy transport measurements derived from optical probing are only included at distances greater than $200 \mu \mathrm{m}$ from the cone tip, where it is safely assumed that any expansion was purely due to the heating along the wire.

The interferometric data was used to create a two-dimensional (2D) density map, assuming that the plasma expansion is approximately cylindrically symmetric. The interferograms were loaded into the IDEA (Interferometrical Data Evaluation Algorithms) software package where 2D phase maps were generated. A program called BASIS ${ }^{14}$ was used to take this phase information and carry out a series of Abel inversions (on the basis of the BASEX method $\left.{ }^{15}\right)$ to produce a $2 \mathrm{D}$ density map $(n(r, z))$. Each line profile drawn at a certain distance from the cone tip gave a radial density profile. The maximum electron density that can be obtained from this measurement is limited to $5 \times 10^{19} \mathrm{~cm}^{-3}$ owing to refraction of the probe light out of the collecting optics.

To model the expansion of the plasma, the 1D lagrangian hydrodynamic code MEDUSA was used in cylindrical geometry ${ }^{16}$. The initial electron temperature was set and the expansion profile calculated as a function of time. A Thomas-Fermi model and ideal-gas equation of state were used for the electrons and ions respectively. The $3.5-\mu \mathrm{m}$-radius $\mathrm{Cu}$ plasma was divided into 500 zones and the flux limit set at 0.1 . The simulation was terminated at $400 \mathrm{ps}$ to match the probe timing. Both the size of the inaccessible region seen in the shadowgrams (see the Supplementary Information) and the density profiles obtained from the interferometry were used to infer the initial plasma conditions.

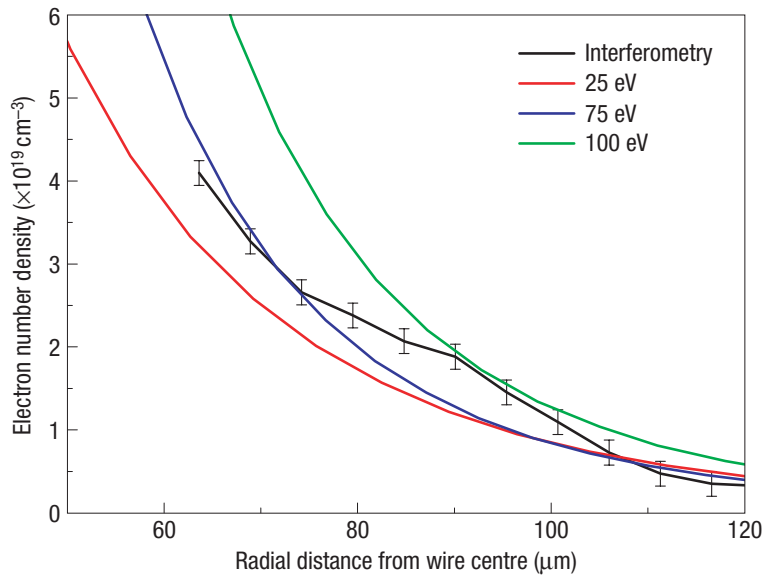

Figure 2 A comparison of computational and experimental radial density

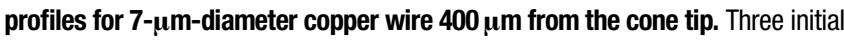
core temperatures are plotted. A surface temperature of $400 \mathrm{eV}$ is assumed in a $0.1 \mu \mathrm{m}$ outer layer of the wire. Error bars for the experimental density profile were calculated from the smallest detectable fringe shift in the interferogram.

It was found that the experimental data could only be reproduced when the outer surface of the wire plasma was heated to a higher temperature than the core during the interaction (see the Supplementary Information).

A series of simulations was carried out using a range of core and surface temperatures for varying surface thicknesses. The radial expansion profile was found to be most sensitive to the initial core temperature. This is shown in Fig. 2, where the experimentally derived radial density profile $400 \mu \mathrm{m}$ from the cone tip is shown together with three simulated profiles using different initial core temperatures. The simulated results were found to best match those obtained experimentally when the outer $0.1 \mu \mathrm{m}$ of the wire was heated to $400 \mathrm{eV}$. The core temperature was $100 \mathrm{eV}$, $75 \mathrm{eV}$ and $25 \mathrm{eV}$ at distances of $200 \mu \mathrm{m}, 400 \mu \mathrm{m}$ and $600 \mu \mathrm{m}$ from the cone tip, respectively. An outer surface that was thinner (thicker) than $0.1 \mu \mathrm{m}$ produced lower (higher) densities than were measured experimentally. It was found that the expansion profiles were relatively insensitive to outer temperatures in the range of $350-450 \mathrm{eV}$. To cross-check these results, simulations were also carried out using the radiation-hydrodynamic code HELIOS-CR ${ }^{17}$, which includes radiation transport, and the results were found to be almost identical. From this comparison, it can be concluded that when using the given range of parameters, radiation transport has little effect.

Previous work into surface transport of electrons during longpulse laser interactions revealed lateral electron motion around the laser focal spot due to crossed electric and magnetic fields ${ }^{18-21}$ ( $E \times B$ forces). However, with the cone-wire geometry this cannot account for longitudinal transport along the wire surface.

Surface transport of fast electrons has been observed in previous PIC energy transport calculations ${ }^{1}$, where the radial electric field balances the azimuthal magnetic field and confines the fast electron flow to the wire surface. However, in those simulations the effect of the return current heating was not modelled accurately owing to the high background temperature. To model the heating of the wire, the implicit hybrid PIC-fluid code LSP ${ }^{22}$ was used. LSP is able to model fast electrons using a PIC description while treating the background plasma as a fluid. The background plasma is modelled using a perfect-gas equation of state with 


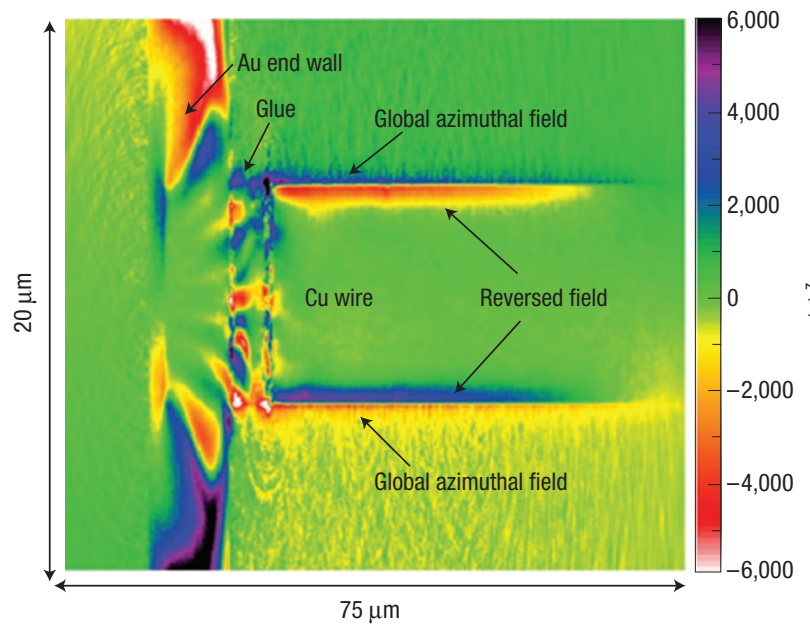

Figure 3 LSP modelling of the azimuthal magnetic field structure at the cone tip, $\mathbf{6 0 0}$ fs after the main interaction. A reversed field can be seen on the inside of the wire surface corresponding to the ohmic return current.

a fixed ionization state. A Spitzer resisitivity model allows the fluid electrons to be heated via the cold return current through ohmic heating.

The end portion of the cone-wire assembly was modelled using a $7-\mu \mathrm{m}$-thick Au slab, with a 8 - $\mu \mathrm{m}$-diameter $\mathrm{Cu}$ wire attached via a $5 \mu \mathrm{m}$ glue joint. The simulation area of $160 \times 30 \mu \mathrm{m}$ was modelled using $800 \times 600$ cells. The glue material was assumed to be fully ionized, whereas the $\mathrm{Au}$ and $\mathrm{Cu}$ were assumed to have +30 and +19 ionization states, respectively. Direct modelling of the laser was not possible with the current version of LSP. Instead the laser was modelled as a relativistic PIC electron beam that was injected into the Au slab with parameters based on the laser pulse $(700 \mathrm{fs}$ pulse duration, $7 \mu \mathrm{m}$ spot size). The longitudinal momentum of the electron beam was given by a relativistic 1D maxwellian with a mean energy calculated from the laser ponderomotive potential. The transverse components were modelled as a $2 \mathrm{D}$ nonrelativistic maxwellian with a mean energy corresponding to a beam divergence angle of $50^{\circ}$.

Figure 3 shows the azimuthal magnetic field $600 \mathrm{fs}$ after the start of the interaction. A global field can be seen on the outside of the wire, corresponding to the net forward current along the surface of the wire. A reversal of the magnetic field can be seen on the inside, resulting from a spatially separated return current at the wire-vacuum interface. The early development of the magnetic field can be seen in the Supplementary Information. The fast electron population that flows along the surface draws the thermal return current within a slightly smaller radius. This results from the requirement to have spatially localized current balance as calculated by Bell et al. ${ }^{23}$. The increased return current in the thin $(\approx 0.2 \mu \mathrm{m})$ surface layer results in a surface temperature of $800 \mathrm{eV}$, double that of the core $(400 \mathrm{eV})$ at a distance of $75 \mu \mathrm{m}$ from the cone tip. The increased filamentation seen in the glue joint in Fig. 3 is a likely explanation for the reduced energy coupling into the General Atomics cone-wire assemblies, particularly as the laser-plasma interaction occurs directly with the glue joint in those targets.

The presence of an enhanced current close to the wire surface may at first seem similar to the classical case of a skin current, but the distribution of the electron population is quite different. The skin effect is seen to occur for the case of an oscillating electron population in a conductor when an electromagnetic field

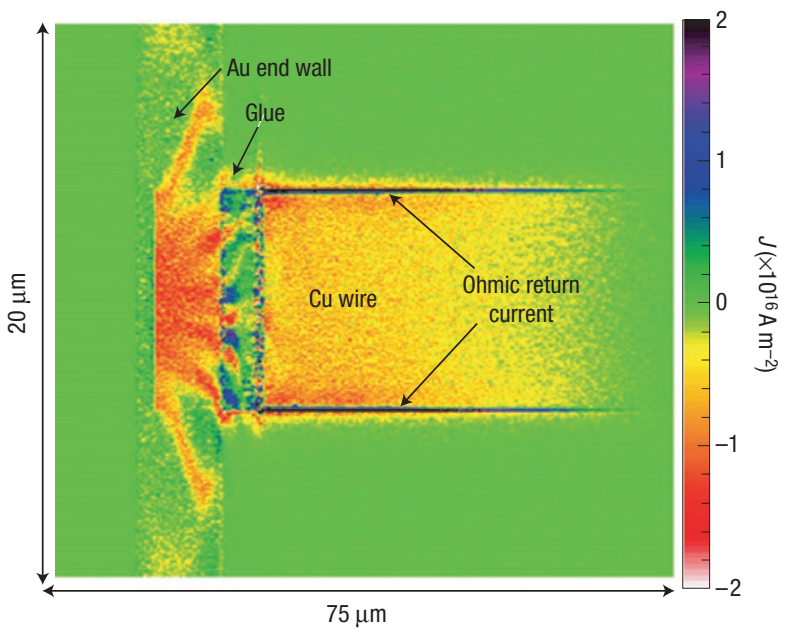

Figure 4 LSP modelling of the net current density inside the wire, 600 fs after the main interaction. The thin layer at the wire surface containing the strong return current can be seen.

is applied ${ }^{24}$. In that case, the current density is almost entirely confined within the skin depth, which is dependent on the material properties and the electromagnetic frequency. In our simulations, fast electrons are seen to propagate throughout the wire, with an enhanced population confined at the surface of the wire. This enhanced population then leads to a greater degree of ohmic heating in a thin outer layer due to the thermal return current. Figure 4 shows the current density inside the wire $600 \mathrm{fs}$ after the main interaction (the current density at earlier times can be seen in the Supplementary Information). Whereas a net forward current can be seen inside the bulk of the wire, a localized return current can clearly be seen at the wire surface.

It may be possible to increase the energy coupling substantially under the experimental conditions reported here by increasing the diameter of the wire while ensuring, as has been demonstrated here, that the glue joint is minimized. In addition, the use of highercontrast-ratio laser pulses could increase the coupling, possibly by changing the wavelength of the heating pulse to $2 \omega_{0}$ (ref. 25). These effects will be the subject of future work.

\section{METHODS}

The experiment was conducted on the Vulcan PetaWatt laser system ${ }^{26}$ at the Rutherford Appleton Laboratory. Vulcan petawatt is a Nd:glass laser that delivered intensities of $4 \times 10^{20} \mathrm{Wcm}^{-2}$ on target at an operating wavelength of $1.054 \mu \mathrm{m} .300 \mathrm{~J}$ of laser energy was delivered in a pulse of $0.7 \mathrm{ps}$ duration, and was focused onto the target using an $f / 3$ parabola to a spot size of $7 \mu \mathrm{m}$. Approximately $30 \%$ of the incident laser energy was contained within the central focal spot.

The expansion profile of the heated targets was diagnosed using an optical probe that was passed transversely across the target surface. The probe beam was created from a small part of the main beam that was frequency-doubled to $2 \omega(527 \mathrm{~nm})$. The probe had a duration of $0.7 \mathrm{ps}$ and was timed to cross the target $400 \mathrm{ps}$ after the main interaction pulse.

In this experiment, a 7 -cm-diameter, $40-\mathrm{cm}$-focal-length achromatic lens was used to collect the probe light after the target. The image was relayed outside the target chamber where the beam was split, providing a shadowgraphy channel and a Nomarski interferometer ${ }^{27}$. An 8-bit CCD (charge coupled device) recorded the shadowgrams, whereas a 16-bit Andor Technology CCD was used for the interferometry measurements. The total $f$-number of the imaging system was $f / 12$ and the magnification was $\times 5$. The spatial resolution was $12 \mu \mathrm{m}$ for both diagnostic channels. 
Gold cone targets, manufactured at Osaka University, had an opening angle of $30^{\circ}$ and had a $7-\mu \mathrm{m}$-thick gold end wall. $1 \mathrm{~mm}$ wires were attached to the cone tip by a glue joint of $<5 \mu \mathrm{m}$. Identical-opening-angle gold cone targets, manufactured at General Atomics, were irradiated. In these cases, the $1 \mathrm{~mm}$ wire targets were embedded so that the wire itself formed the end wall of the cone and the end wall around the wire was sealed with a glue joint. In that case, the electrons had to traverse $5 \mu \mathrm{m}$ of glue and $10 \mu \mathrm{m}$ of copper before emerging from the end of the cone. The copper wires were $10 \mu \mathrm{m}$ and $20 \mu \mathrm{m}$ in diameter.

\section{Received 24 April 2007; accepted 18 September 2007; published 28 October 2007.}

\section{References}

1. Kodama, R. et al. Plasma devices to guide and collimate a high density of $\mathrm{MeV}$ electrons. Nature 432 1005-1008 (2004)

2. Kodama, R. et al. Fast heating of ultrahigh-density plasma as a step towards laser fusion ignition. Nature 412, 798-802 (2001).

3. Kodama, R. et al. Nuclear fusion-fast heating scalable to laser fusion ignition. Nature 418 , 933-934 (2002).

4. Tabak, M. et al. Ignition and high gain with ultrapowerful lasers. Phys. Plasmas 1, 1626-1634 (1994).

5. Norreys, P. A. et al. Experimental studies of the advanced fast ignitor scheme. Phys. Plasmas 7, $3721-3726$ (2000).

6. Gremillet, L. Bonnaud, G. \& Amiranoff, F. Filamented transport of laser generated relativistic electrons penetrating a solid target. Phys. Plasmas 9, 941-948 (2002).

7. Silva, L. O., Fonseca, R. A., Tonge, J. W., Mori, W. B. \& Dawson, J. M. On the role of the purely transverse Weibel instability in fast ignitor scenarios. Phys. Plasmas 9, 2458-2461 (2002).

8. Tatarakis, M. et al. Propagation instabilities of high intensity laser-produced electron beams. Phys. Rev. Lett. 90, 175001 (2003).

9. Wei, M. S. et al. Observations of the filamentation of high intensity laser-produced electron beams Phys. Rev. E 70, 056412 (2004)

10. Bret, A., Firpo, M. C. \& Deutsch, C. Characterisation of the initial filamentation of a relativistic electron beam passing through a plasma. Phys. Rev. Lett. 94, 115002 (2005).

11. Jung, R. et al. Study of electron beam propagation through pre-ionized dense foam plasmas. Phys. Rev. Lett. 94, 195001 (2005).

12. Lancaster, K. L. et al. Measurements of energy transport patterns in solid density laser plasma interactions at intensities of $5 \times 10^{20} \mathrm{~W} \mathrm{~cm}^{-2}$. Phys. Rev. Lett. 98, 125002 (2007).

13. Key, M. H. et al. Study of electron and proton isochoric heating for fast ignition. J. Physique IV 133 , 371-378 (2006).

14. Gregory, C. Astrophysical Jet Experiments with Laser-Produced Plasmas. Thesis, Univ. of York (2007).
15. Dribinski, $V$ et al. Reconstruction of abel-transformable images: The Gaussian basis-set expansion abel transform method. Rev. Sci. Instrum. 73, 2634-2642 (2002).

16. Christiansen, J. P., Ashby, D. E. T. F. \& Roberts, K. V. MEDUSA—one-dimensional laser fusion code Comp. Phys. Comm. 7, 271-287 (1974).

17. MacFarlane, J. J., Golovkin, I. E. \& Woodruff, P. R. HELIOS-CR-A 1-D radiation-magnetohydrodynamics code with inline atomic kinetics modeling. J. Quant. Spectrosc. Radiat. Transfer 99, 381-397 (2006)

18. Yates, M. A. et al. Experimental-evidence for self-generated magnetic-fields and remote energy deposition in laser-irradiated targets. Phys. Rev. Lett. 49, 1702-1704 (1982).

19. Amiranoff, F. et al. The evolution of two-dimensional effects in fast-electron transport from high-intensity laser plasma interactions. J. Phys. D 15, 2463-2468 (1982).

20. Forslund, D. W. \& Brackbill, J. U. Magnetic-field-induced surface transport on laser-irradiated foils. Phys. Rev. Lett. 48, 1614-1617 (1982).

21. Fabbro, R. \& Mora, P. Hot-electrons behavior in laser-plane target experiments. Phys. Lett. A 90, $48-50$ (1982).

22. Welch, D. R., Rose, D. V., Oliver, B. V. \& Clark, R. E. Simulation techniques for heavy ion fusion chamber transport. Nucl. Instrum. Methods Phys. Res. A 464, 134-139 (2001).

23. Bell, A. R., Robinson, A. P. L., Sherlock, M., Kingham, R. J. \& Rozmus, W. Fast electron transport in laser-produced plasmas and the KALOS code for solution of the Vlasov-Fokker-Planck equation. Plasma Phys. Control. Fusion 48, R37-R57 (2006).

24. Jackson, J. D. Classical Electrodynamics Ch. 5, 219-221 (Wiley, Hoboken, 1999).

25. Baton, S. et al. Recent experiments on electron transport in high intensity laser matter interaction Plasma Phys. Control. Fusion 47, B777-B789 (2005).

26. Danson, C. N. et al. Vulcan Petawatt-an ultrahigh interaction facility. Nucl. Fusion 44, S239-S246 (2004).

27. Benattar, R., Popovics, C. \& Sigel, R. Polarized-light interferometer for laser fusion studies. Rev. Sci. Instrum. 50, 1583-1585 (1979).

\section{Acknowledgements}

This work was supported by the UK Engineering and Physical Sciences Research Council and the Science and Technology Facilities Council. American colleagues acknowledge support from the US Department of energy contract number W-7405-Eng-48. Japanese colleagues acknowledge the Japan Society for the Promotion of Science. The authors gratefully acknowledge the support of the staff of the Central Laser Facility.

Correspondence and requests for materials should be addressed to P.A.N.

Supplementary Information accompanies this paper on www.nature.com/naturephysics.

Author contributions

F.N.B., R.R.F., J.S.G., M.H.K., R.K., K.L.L., P.A.N., R.S. and L.V.W. were all involved in project planning. J.S.G. and P.A.N. carried out the data analysis and wrote the letter. All other named authors contributed to the experimental work and corrected the manuscript.

Reprints and permission information is available online at http://npg.nature.com/reprintsandpermissions/ 